\title{
Digital Construction of Large-Size Minimal Surface: Transformation from Mathematical Model to Mechanical Model
}

\author{
Chaohao SU, a,c, 1 , Shujian ZHANG ${ }^{\mathrm{a}}$ and Yi FAN ${ }^{\mathrm{a}}$ \\ a School of Architecture, South China University of Technology, Guangzhou, China \\ ${ }^{\mathrm{b}}$ Architectural Design and Research Institute of SCUT Co., Ltd, Guangzhou, China \\ c State Key Laboratory of Subtropical Building Science, China
}

\begin{abstract}
Under the background of digitization, the basic algorithm of minimal surface provides novel and rich creative themes for architectural design. However, due to the high complexity and extreme thinness, how to convert the mathematical model to the mechanical model has become the biggest challenge in the application of large-size minimal surface. On the basis of analysing the relation between force and form, this paper explores and verifies the concept and method of transforming mathematical model into mechanical model by combining qualitative analysis and quantitative calculation. Combined with a work named "Yunjian Linglong" and the related experiments, it provides theoretical clues for the technical route of highly complex heteromorphic hyperboloid, and empirical reference value for the cooperation between shape design and structural design under the digital background.
\end{abstract}

Keyword. Digital construction, minimal surface, structural form-finding, ultra-thin shell.

\section{Introduction}

In the field of mathematics, minimal surfaces are surfaces with mean curvature equal to 0 [1], that is, a stable surface with minimum surface area can be formed under certain constraints. It has been a significant research direction and has attracted scholars in many fields since Lagrange first put forward the minimal surface equation in 1774 [2]. Due to its unique mathematical beauty and highly complex and changeable spatial characteristics, minimal surfaces gradually began to attract the attention of architects. German Frei Otto was the first architect to explore membrane structure and study the minimum area of membrane structure expression on buildings [3]. The Stadium of Munich Olympic Sports Center in Germany was derived from his research on the structural form-finding of the minimally curved membrane [4]. Sergio Musmeggi designed the Balsanto Viaduct by extending the minimal surface to the practical engineering of compressive concrete construction employing soap film and rubber cloth physical form-finding method.[5] While both the Taichung Metropolitan Opera

\footnotetext{
${ }^{1}$ Corresponding Author, Chaohao SU, School of Architecture, South China University of Technology, Guangzhou, China; Architectural Design and Research Institute of SCUT Co., Ltd, Guangzhou, China; State Key Laboratory of Subtropical Building Science, China; Email: 11083812@qq.com.
} 
constructed by Toyo ITO and Balmond and the Winton Gallery by Zaha Architects use the nonlinear nature of minimal surfaces to create a unique architectural space [6].

With the advancement of digital design and CNC technology, it is possible to realize the complex irregular building shapes by means of digital production tools. MARC FORNES's research and the $6 \mathrm{~mm}$-thick Maxima giant device provide a huge thrust to the lightness of minimal surfaces [7]; Knit Candela, designed by the Federal Institute of Technology of Zurich and Zaha Hadid Architects, introduced an innovative minimal surface construction method for 3D braided shells [8]. Domestic research on minimal surfaces has made some breakthroughs in recent years. The FLORA series, led by Jiong $\mathrm{Xu}$ of Nanjing University of the Arts, has made some breakthroughs in the construction of minimal surfaces through the process of form-finding, mechanical simulation, structural optimization, node design and full-scale construction [9]; the anthozoan space installation, designed by architect Zhenfei Wang at FUN, combined with children's activities to create rich urban Spaces by means of spatial continuity of minimal surfaces [10]. (figure 1)

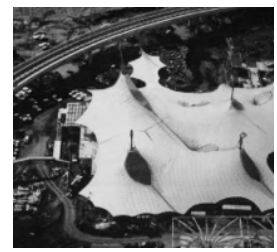

Munich Central Stadium.

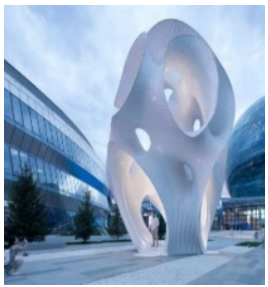

Maxima device.

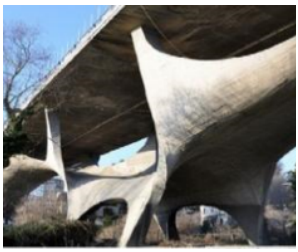

Pasanto Viaduct.

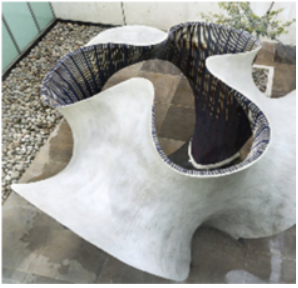

Knit Candela.

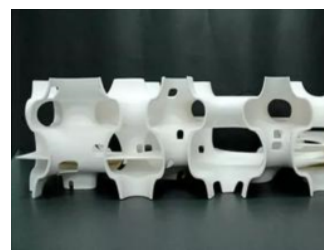

Taichung Metropolitan Opera.

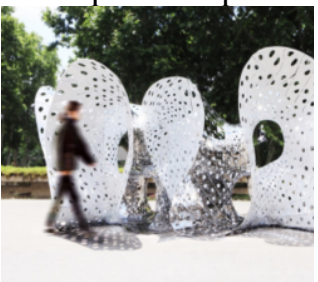

"FLORA" series.

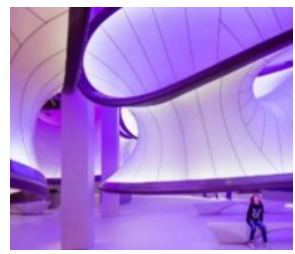

Winton Gallery.

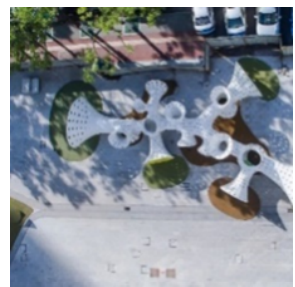

The anthozoan

space

installation.

Figure 1. The practices of minimal surface.

From the above development of minimal surface and the latest research results in recent years, it can be seen that the development of minimal surface in the field of architecture is still in a relatively primary stage, and it still has great research potential and development value in both the design stage and construction stage. Due to the rich spatial form of minimal surfaces, how to realize the transformation of the mathematical model to the mechanical model under the actual working conditions is the critical problem for the large-size application and development of minimal surfaces. 


\section{Experimental Methods}

\subsection{Algorithm Optimization - Based on the Basic Formation Method of Minimal Surface}

The simulation of the basic algorithm of minimal surface is completed through the setting of Rhino and Grasshopper programs (figure 2). The algorithm is to create a certain number of three-dimensional equational points within a Box range, determine the range of Iso Surfaces by the minimal surface expressions, and then use the Iso Surface algorithm to fit Iso Surfaces in the form of mesh. As shown in figure 3, the complex form of minimal surface can be changed according to different mathematical expressions, and simple basic parameters, such as the period, can be adjusted to obtain complex and logical spatial form. However, the pattern obtained by the basic algorithm is relatively fixed. It is difficult to meet the demands of diverse space, even if forms of different density distributions are obtained by adjusting the calculation period. Therefore, it is necessary to improve the basic algorithm: The points in the space are turned into controllable points, then the algorithms and procedures of these points are superimposed. By adjusting various parameters: the position, number, threshold adjustment and other relevant parameters of points, digital information visualization, fast simulation and coordination are realized to finally achieve the goal of optimization.

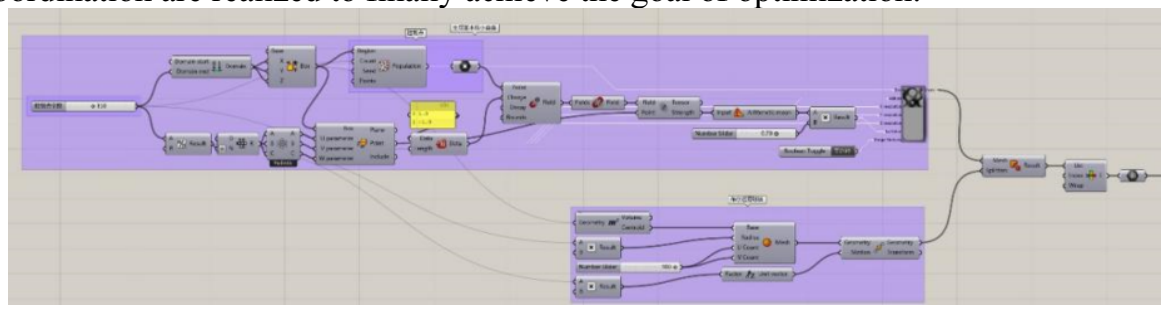

Figure 2. The improved algorithm based on basic algorithm of minimal surface.
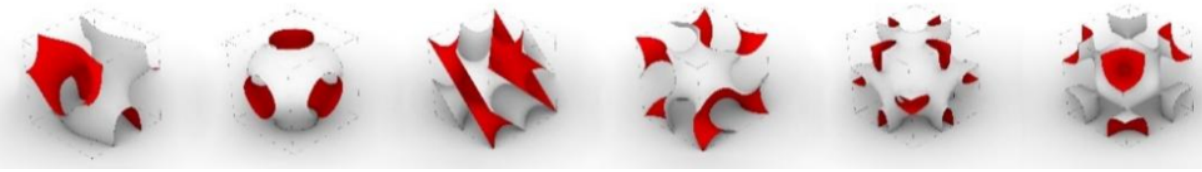

Figure 3. Minimal surfaces under different mathematical expressions.

\subsection{Structural Form-Finding: Transformation from Mathematical Model to Mechanical Model}

Minimal surfaces are generated by mathematical algorithms which are purely mathematical models, not mechanical models in a physical environment. Therefore, under mechanical conditions, pure mathematical models need to be deformed and transformed into mechanical models, aiming to create high-performance, highly adaptable and dynamic structural systems, and finally achieve the unity of force and form. In terms of technical operation, the mathematical model generated by the basic algorithm is set in a schedulable state by means of digital design, and the transformation of the mechanical model from mathematical model is realized by adjusting the parameters related to the mechanical rules. At the same time, the rationality of the morphological rationality is evaluated and fed back through the repeated evaluation and optimization of the mechanical structure software (figure 4 and 5). 


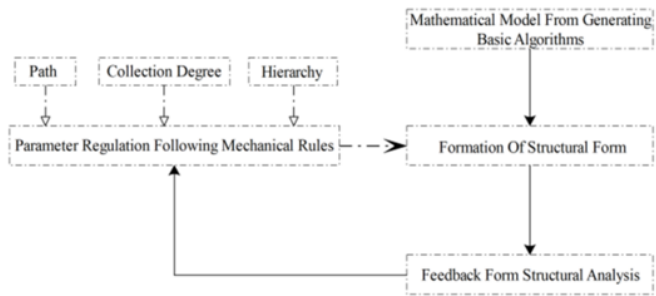

Figure 4. Algorithm optimization.

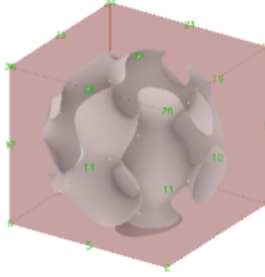

The homogeneous lattice forms minimal surface.

Figure 5. Different lattice forms minimal

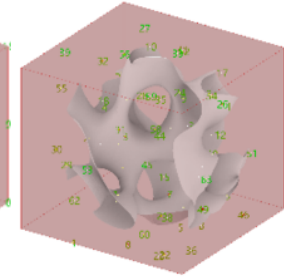

Control points forms minimal surface. surfaces.

\subsubsection{Transformation to Tubular Objects}

In the traditional Cartesian Orthogonal System, the standard method to obtain a more extensive span is arching, which resists massive lateral thrust through larger materials on both sides of the arch foot. In this way, the transfer loop of force flow inside the material is formed by adjusting the shape to form a ring. When the force in one direction changes, the force in the other direction will resist it, showing features of spatial resistance. I On the technical route, by moving the position parameters of control points, more barrel shapes are generated to realize the force flow loop, thus forming a self-balancing system, achieving higher overall efficiency and lower material internal consumption.

\subsubsection{Transformation to Tree Frame}

Unlike the beam-column structural system in cartesian orthogonal coordinates, the minimal surface model structural system is a three-dimensional spatial resistance heterometric system, that is, structural elements generate overall spatial stiffness through the interaction between each direction of space. On the technical route, through the program regulation, that is, by moving the position parameters of control points, the tree frame system is formed to adjust the aggregation and dispersion of force flow to realize the diversity of forms. A tree-shaped branch is a typical method to reduce the span by changing the stress concentration. Under the same external conditions, the single section of tree-shaped pillar will be significantly smaller, and the branches also significantly improve the bearing capacity of the whole structure.

\subsubsection{Transformation to a Concentrated State from a Homogeneous State}

The minimal surface model generated through the program does not consider the load, mechanical distribution and other factors, and the generated model is usually relatively homogeneous and foamy, which is not conducive to force flow transfer. From the point of view of force flow hierarchy, better mechanical properties can be obtained by increasing the level of force transmission. Through the program regulation, the position parameters of points, the vertical load accumulates from small to large the from top to bottom. At the same time, the burden of a single level can be reduced by adding layers. All internal components pull and transfer forces to each other, weakening the hierarchical order to adapt to a more complex spatial structure. 


\subsubsection{Connecting node design}

The fabrication method is to expand into multiple components through stripes, so the construction design between each unit type components is crucial. Buckling and instability are the disadvantages of thin-walled structure, so the strengthening of out-ofplane stiffness is an important aspect that must be considered. It is an important idea to strengthen stiffness through detail structure without increasing thickness.

Overlap each other to form a local rigid domain. The connection between two adjacent strip elements can be designed as an overlapping ear plate, with two rows of rivets arranged above the ear plate at a certain distance (the distance between the rivets is a moment arm). The overlapping part between the rivets forms a local rigid domain, which effectively resists the out-of-plane bending deformation. The zipper effect: a potential stiffener. The ear plate is arranged along the edge of the strip, forming a interlocked zipper effect to act as a stiffener inside the shell unit. With the density change of these chains, the out-of-plane stiffness of the minimal curved shell can be adjusted.

\section{Experiments and Results}

At the same time of adjusting algorithm parameters, each state model in the transformation process was input into the structural calculation software ABAQUS for preliminary static calculation. The experiment selects the appropriate element component as the physical experiment object. The structure analysis uses the software ABAQUS and uses the shell element (SR3) to simulate the aluminum sheet. The aluminum's density $\rho=2.7 \times 10^{-9} \mathrm{t} / \mathrm{mm} 3$, the elastic modulus $\mathrm{E}=69000 \mathrm{MPa}$, and the Poisson's ratio $\mathrm{v}=0.3$. The applied uniform load is $10-100$ times the self-weight, and the boundary condition is to constrain the translational freedom of the three directions of the ground contact interface, so as to evaluate the influence and development trend of this transformation process on the overall structural performance, and prove whether the digital structure form-finding method in this paper is feasible.

\subsection{Mechanical Test of Hoop Effect}

This paper verifies the positive effect of the tubular objects on the overall spatial resistance through simple mechanical experiments. Three groups were set up for experiment comparison. In a space with a basic shape of $6 \mathrm{~m} \times 6 \mathrm{~m} \times 6 \mathrm{~m}$, completely closed tubes are formed respectively, as well as connectors with different degrees of closure. The observation will examine whether forming a wholly enclosed tube on the overall structure can withstand the gravity load's stress and displacement. As shown in figure 6, it can be found that under the same experimental conditions, the maximum stress of tubular objects is $5.535 \times 10^{7} \mathrm{~Pa}$, and the maximum displacement is $0.1218 \mathrm{~m}$. The maximum stress of incomplete tubular objects is $5.198 \times 10^{9} \mathrm{~Pa}$ and $5.121 \times 10^{9} \mathrm{~Pa}$, and the maximum displacement is $7.605 \mathrm{~m}$ and $6.000 \mathrm{~m}$, respectively. Through the comparison of figure. 6 and the above data, it can be found that the tubular objects have greatly improved the overall mechanical properties, and the hoop effect is noticeable. Although the closure degree is different in the two groups of not completely closed experiments, the stress and displacement change little. In summary, tubular objects are the main factors affecting the overall spatial resistance. 


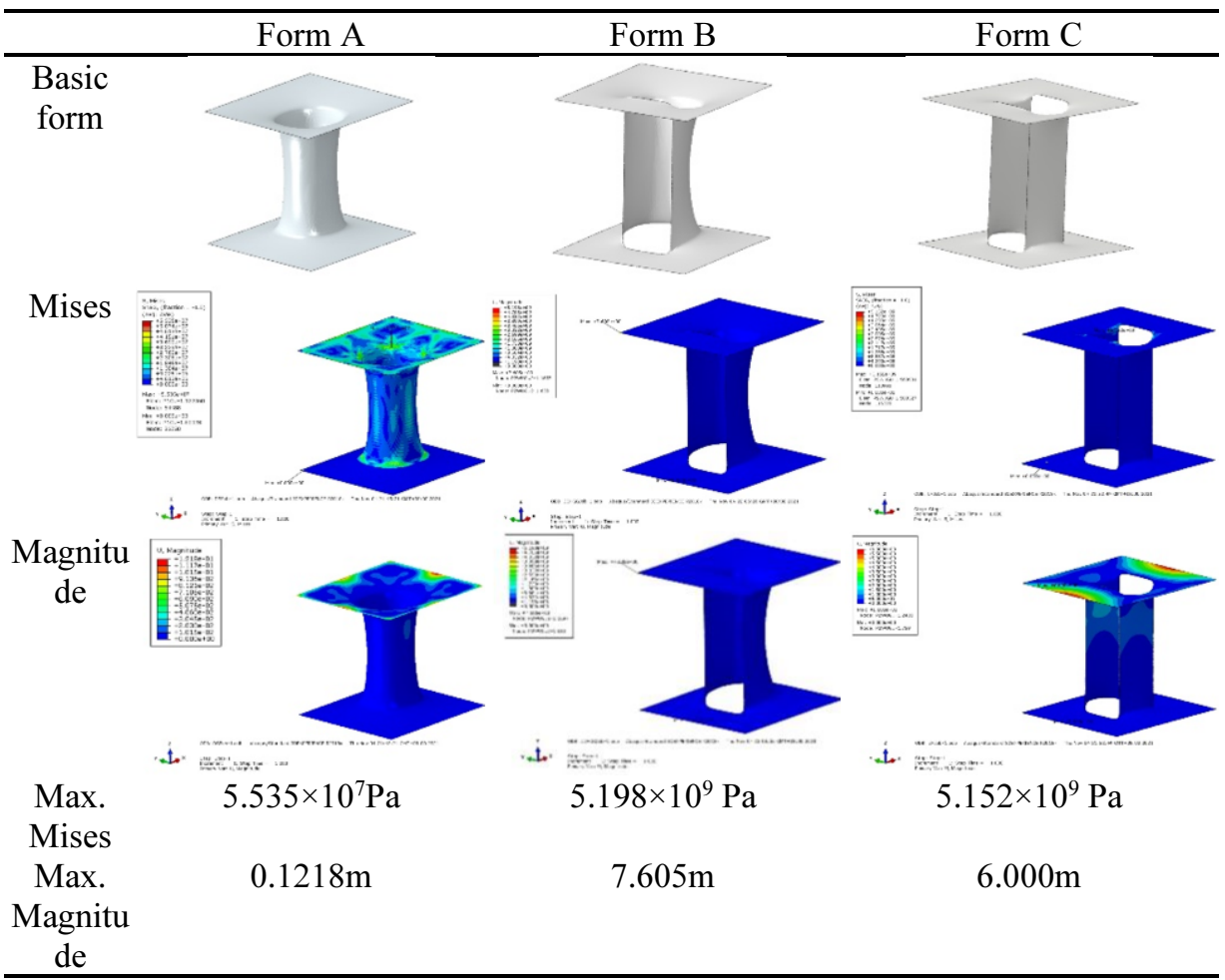

Figure 6. Different lattice forms minimal surfaces.

Through simple mechanical tests (figure 7), the transfer paths of circumferential and vertical principal stresses can be clearly seen by observing the force flow distribution. The force flow loop is formed through the circumferential constraint, and the spatial resistance is formed. Although the shape slightly changes from the intact tubular object to the slightly deformed tubular object, the hoop stress still exists. This deformed shape provides a continuous possibility for creating a rich spatial form.

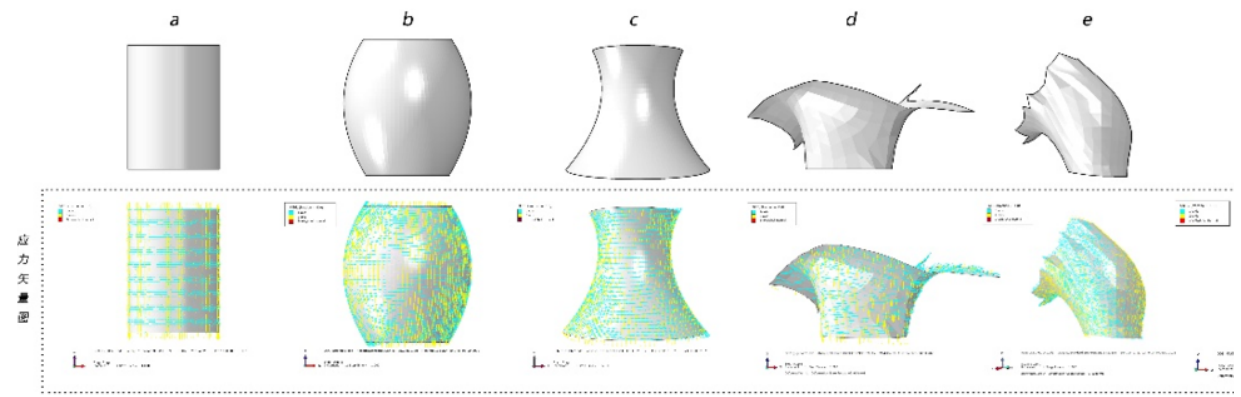

Figure 7. Verification of hoop effect.

\subsection{Mechanical Test of Tree Structure}

This paper verifies the positive effect of the formation of tree structure on the overall spatial resistance through simple mechanical experiments. In the experiment, two groups 
were set up for comparison, which are single cylindrical structure and branch tree structure. Observe the overall structural stress and displacement values under gravity load. As shown in figure 8 , it can be found that under the same experimental conditions, the maximum stress of a single cylindrical structure is $1.744 \times 10^{7} \mathrm{~Pa}$, and the maximum displacement value is $0.2085 \mathrm{~m}$. The maximum stress of the branch tree structure is $1.564 \times 10^{7} \mathrm{~Pa}$ and $1.214 \times 10^{7} \mathrm{~Pa}$, and the maximum displacement value is $0.1344 \mathrm{~m}$ and $0.1327 \mathrm{~m}$. Through the comparison of figure 8 and the above data, it can be found that the number and location relationship of the bearing structure have a certain impact on the spatial resistance of the overall structure; the force flow in the tree structure is obviously distributed through the tree branch, and the overall maximum stress and displacement are significantly reduced.

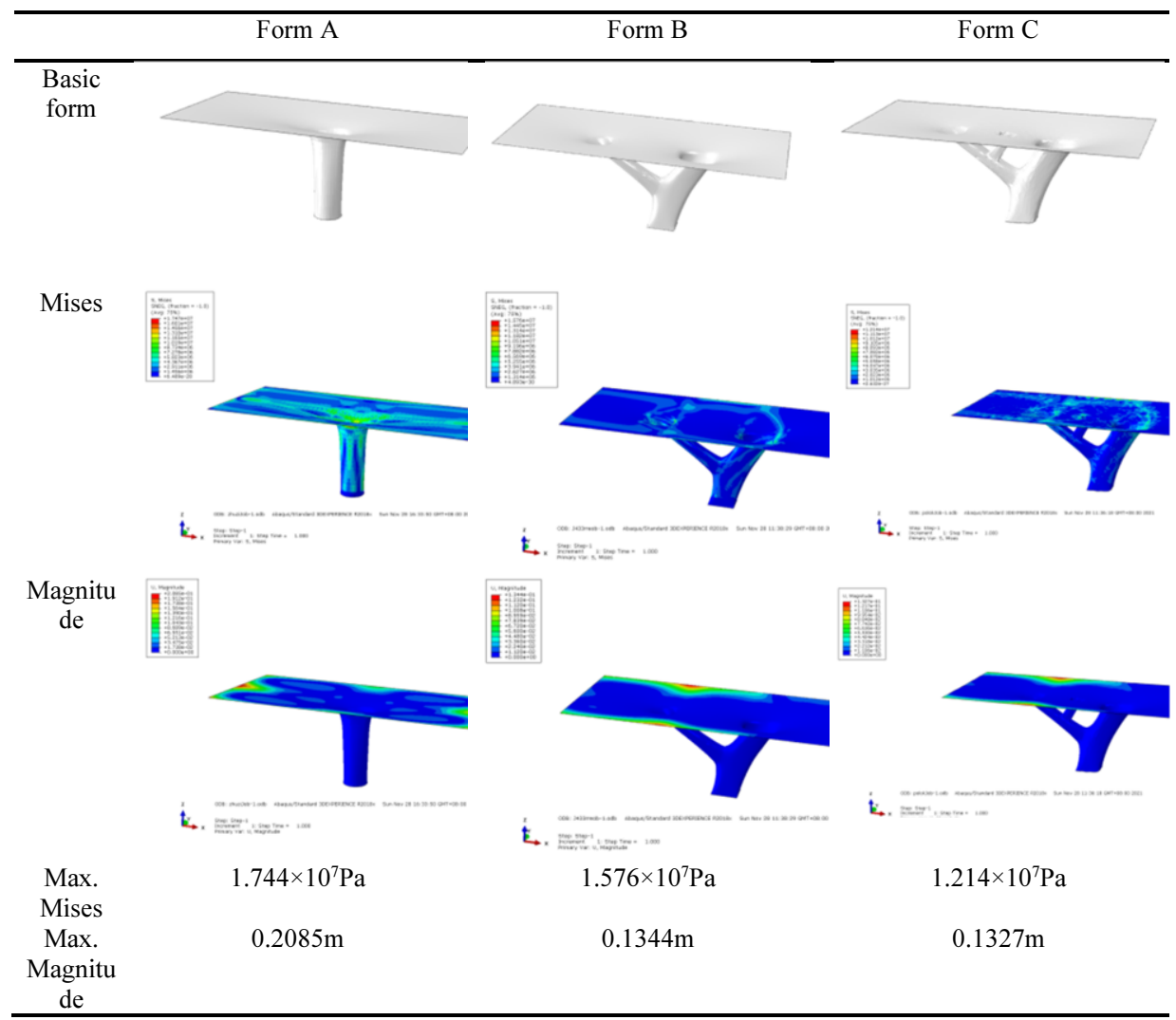

Figure 8. Verification of hoop effect.

\subsection{Mechanical Test of Homogeneous Transformation to Agglomeration.}

This paper verifies the positive effect of the transformation from homogeneity to agglomeration on the overall spatial resistance through simple mechanical experiments. In the experiment, three groups were set up for comparison, which are set up, respectively, under the same mechanical conditions, the influence of homogeneous morphology and agglomeration morphology on the overall stress value and force flow distribution. As shown in figure 9, it can be found that under the same experimental 
conditions, the maximum stress of the homogeneous state is $7.224 \times 10^{8} \mathrm{~Pa}$, the maximum displacement value is $2.907 \mathrm{~m}$, the maximum stress of the agglomeration state is $3.898 \times 10^{7} \mathrm{~Pa}$, and the maximum displacement value is $0.3521 \mathrm{~mm}$. Through the comparison of figure 9 and the above data, it can be found that compared with the homogeneous state, the size of the spatial form formed in the agglomeration state varies greatly, and it can adapt to more complex spatial forms; compared with the homogeneous state, the force flow in the concentrated state is transmitted from the end to the main frame, and the hierarchy is more significant.

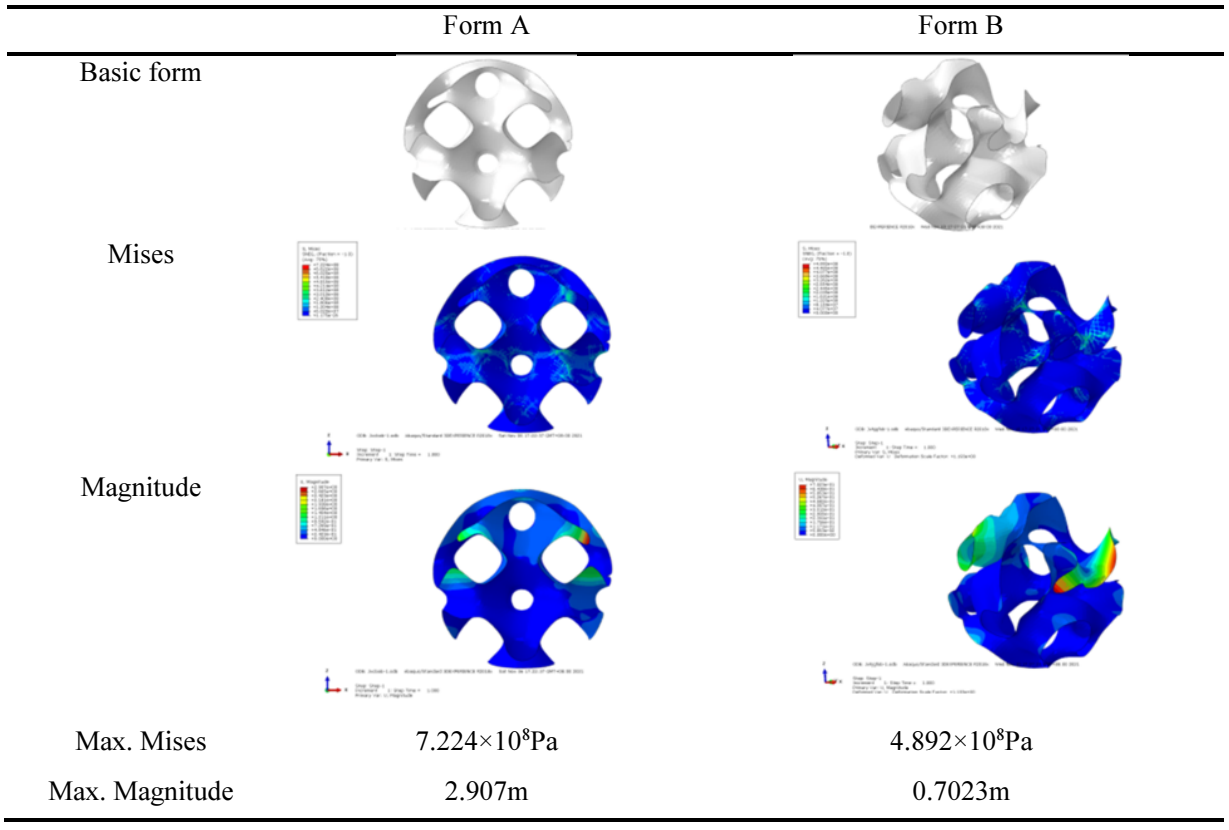

Figure 9. The minimal surface model is generated by different lattice.

\subsection{Mechanical Test of Connection Joints}

This paper verifies the positive effect of tree structure on overall spatial resistance by simple mechanical experiments. In this paper, two groups of experiments were set up to study the influence of the overall stress value and force flow distribution, whether the overlapping ear plates are formed in plane-shape and arch shape respectively, under the same mechanical conditions. As shown in figure 10, it can be found that under the same experimental conditions, the maximum stress of the non-overlapping ear plate is $6.292 \times 10^{6} \mathrm{~Pa}$ and $2.128 \times 10^{8} \mathrm{~Pa}$, and the maximum displacement value is $2.788 \times 10^{-2} \mathrm{~m}$ and $7.790 \times 10^{-4} \mathrm{~m}$. The maximum stress of the overlapping ear plate is $2.430 \times 10^{6} \mathrm{~Pa}$ and $1.755 \times 10^{8} \mathrm{~Pa}$, and the maximum displacement value is $1.302 \times 10^{-3} \mathrm{~m}$ and $6.326 \times 10^{-4} \mathrm{~m}$. Through the comparison of figure 10 and the above data, it can be found that there is an obvious stress concentration phenomenon in the form of overlapping ear-plates structure, which forms a local rigid domain and effectively resists the bending deformation outside the plane. The maximum stress and displacement of the structure with overlapping earplates are significantly reduced, and the local structure enhances the out-of-plane stiffness of the thin shell. 


\begin{tabular}{|c|c|c|c|c|}
\hline & $\mathbf{a}$ & $\overline{a^{\prime}}$ & b & $\mathbf{b}^{\prime}$ \\
\hline \multicolumn{5}{|l|}{ Basic form } \\
\hline \multicolumn{5}{|l|}{ Mises } \\
\hline \multirow{2}{*}{\multicolumn{5}{|c|}{ Magnitude }} \\
\hline & & & & \\
\hline & $2 x-2$ & 2. & 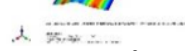 & i $2 x=$ \\
\hline Max. Mises & $6.292 \times 10^{6} \mathrm{~Pa}$ & $2.430 \times 10^{6} \mathrm{~Pa}$ & $2.128 \times 10^{8} \mathrm{~Pa}$ & $1.755 \times 10^{8} \mathrm{~Pa}$ \\
\hline Max. Magnitude & $2.788 \times 10^{-2} \mathrm{~m}$ & $1.302 \times 10^{-3} \mathrm{~m}$ & $7.790 \times 10^{-4} \mathrm{~m}$ & $6.326 \times 10^{-4} \mathrm{~m}$ \\
\hline
\end{tabular}

Figure 10. Verification of ear plate function.

\section{Case}

The work named Yunjian Linglong, designed for Guangzhou International Lighting Festival in 2019 (figure11), is a shell structure of minimal surface generated by mathematical formulas and computer programs. On the one hand, it has a free form and is not confined to a simple orthogonal system, creating a special spatial experience. On the other hand, the aluminium plate with a thickness of only $1.5 \mathrm{~mm}$ supports a $7.8 \mathrm{~m}$ in diameter and height, with no redundant skeleton support and only a thin surface structure like paper. It is also a unique mechanical research work transformed from mathematical space to physical space.
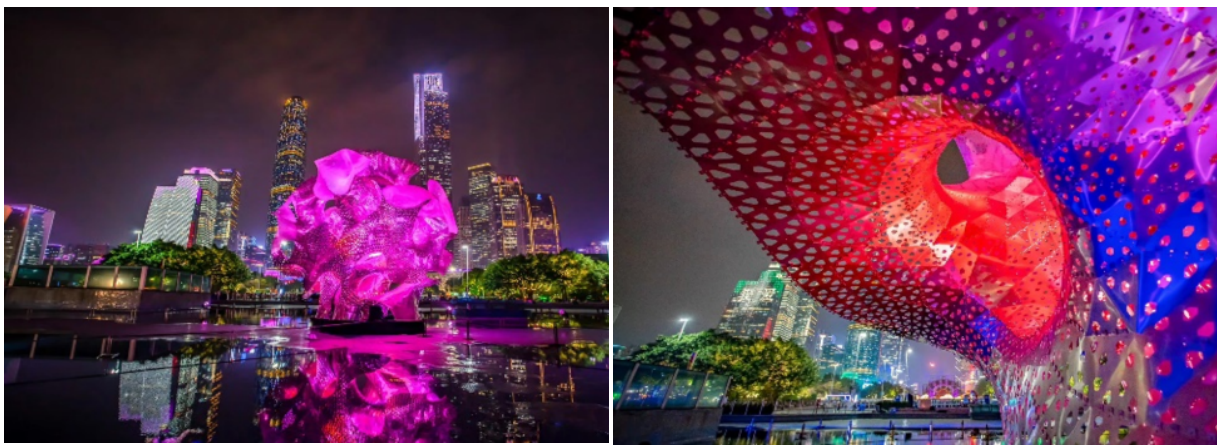

Figure 11. 'Yunjian Linglong' designed for Guangzhou International Light Festival

Rhino and Millipede complete the basic shape design and optimization design of ' Yunjian Linglong '. The mechanical evaluation of mathematical models in different states is carried out by combining structural analysis with parametric design, so as to realize the transformation from mathematical model to mechanical model. Firstly, the dot-matrix randomly generates the control points. Secondly, the original minimal surface model is generated by the dot matrix parametric generation method. Finally, the 
structural form-finding design of the mathematical model is carried out by adjusting the parameters, such as the location of control points, influence factors and so on, so that the model generates more tubular objects and gradually forms the spatial state of multiple tubular frames from the state of homogeneous foam. Each state of the above transformation process is input into the ABAQUS for preliminary static analysis to evaluate the influence of the overall structural performance and development trend of this transformation process (figure. 12)

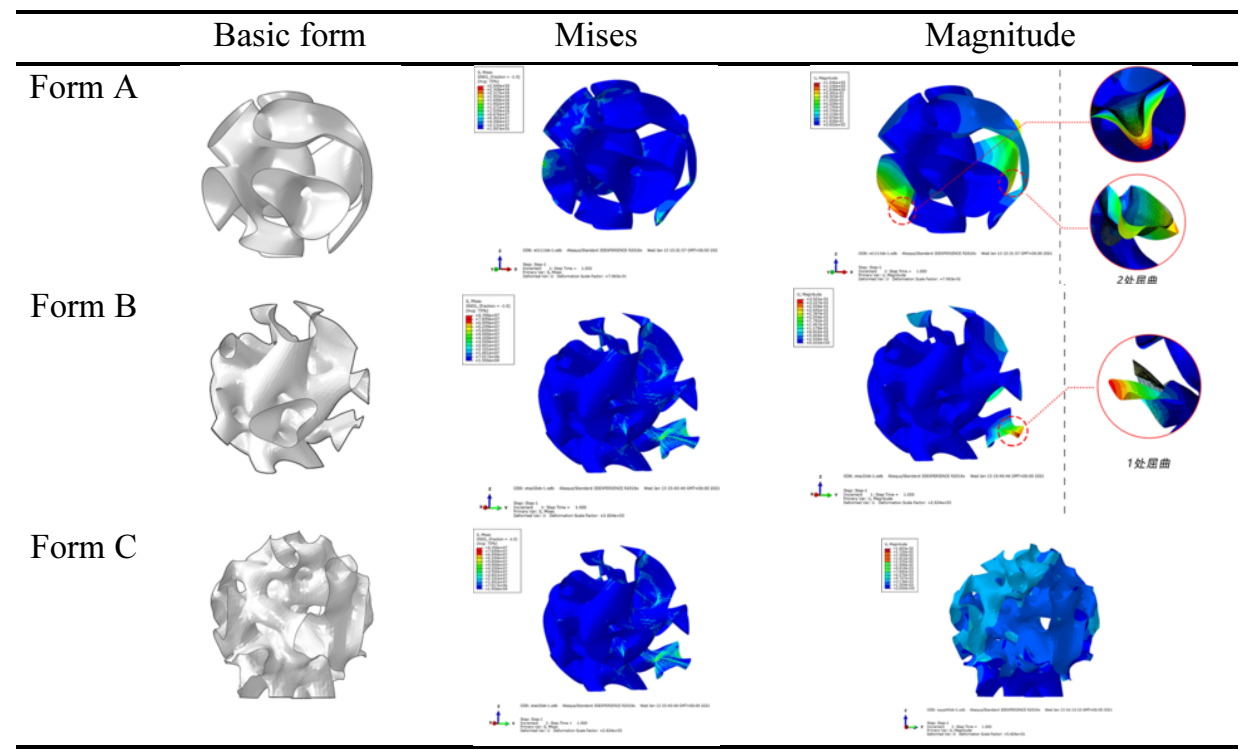

Figure 12. Design optimization process and structural performance verification.

In terms of construction, irregular hyperboloid can create a smooth free-form surface form. This smoothness and freedom have brought significant challenges to actual construction. The following fitting principle is proposed for construction: Firstly, the irregular surface that cannot be expanded into a plane is meshed and divided into surfaces composed of multiple connected meshes. The more units, the smoother the surface; secondly, these finite elements are planarized, in order to be quickly cut by CNC technology. Finally, the plane element is bent and assembled to fit the target surface.

The entire construction process involves the following four steps (figure 13):

1. Geometric subdivision optimization. The heterogeneous meshed model is discretely expanded. Under the programming of grasshopper, the expansion length is divided according to the size requirements of $\mathrm{CNC}$ machine, and form strips.

2. Precise control of planarity. The model information, such as shape, size and so on, is presented on two-dimensional drawings through digital tools. The processed components are typewritten on the $\mathrm{CAD}$ for accurate cutting of $\mathrm{CNC}$ machine tools.

3. Unit surface fitting. The plane with marked angles is bent to fit the unit surface, and the rivets are used to cross the reserved round hole of the ear plate to fix and combine the two units to restore the whole.

4. Overall assembly. Firstly, the 37 groups of units are pre-assembled at low altitude. Then, the unit of tubular frame structure is assembled to produce a certain temporary spatial stiffness, and the outer secondary structure is assembled based on the tubular frame. Finally, the crane or gantry is used for the high-altitude part of the operation. 


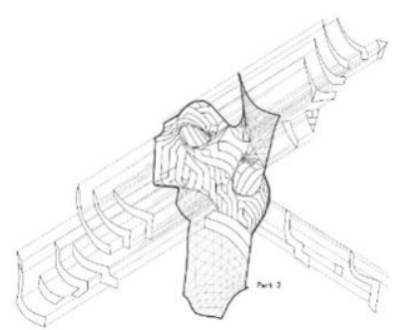

1. Geometric subdivision optimization.

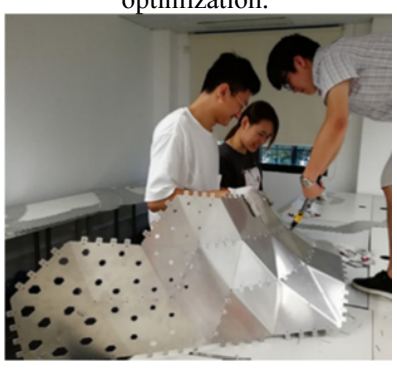

3. Unit surface fitting.

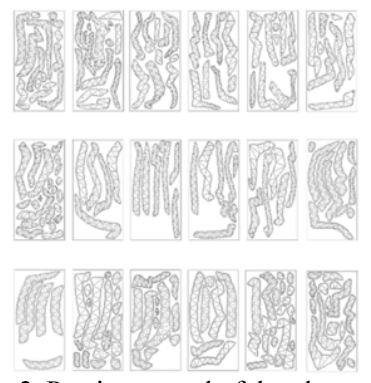

2. Precise control of the plane.

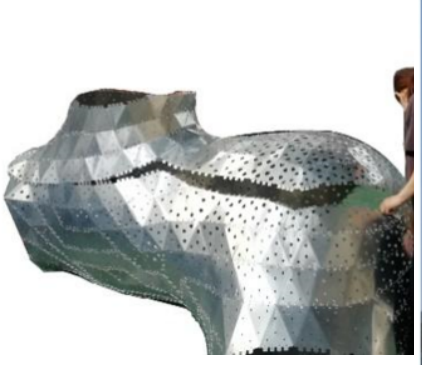

Figure 13. Fabrication process.
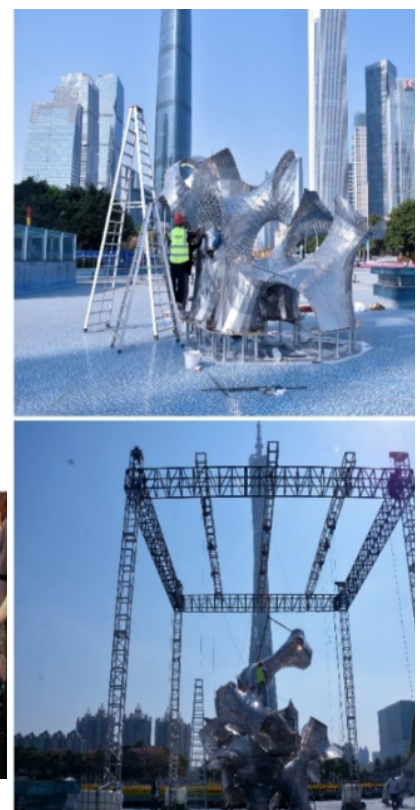

4. Overall assembly

\section{Conclusions}

Digital construction of large minimal surfaces is a frontier research subject in architecture. With the aid of algorithm technology and digital design tools, this paper qualitatively analyzes the experimental method of transforming mathematical space of minimal surface to physical space of reality. By optimizing and adjusting the basic algorithm of the minimal surface, the model is in a schedulable state. Combined with the structural analysis, four directions of structural form-finding are proposed: transforming to tubular structures, transforming to tree-shaped frameworks, and transforming from homogeneous to clustered states, and connecting node design. At the same time, four groups of digital simulation mechanics experiments are designed. The preliminary static quantitative calculation is carried out with the structural calculation software ABAQUS to verify the feasibility of the experimental method in the exploration of the digital construction of the minimal surface. It shows that structure can be a precondition for complex spatial form, not just a constraint. The later structural design can be incorporated into the earlier architectural form-finding process to better promote the seamless connection of the construction process. The project of "Yunjian Linglong " combined the formation logic, structural performance optimization and digital construction to verify the experimental method proposed in this paper.

\section{Acknowledgments}

The research described in this paper is supported by the Open Project Fund of State Key Laboratory of Subtropical Building Science (2017ZB13), and the Research and 
Development Projects of Housing and Urban Rural Development of Guangdong Province (2021-K1-121149). The corresponding author is Chaohao Su.

\section{References}

[1] Hao Yongxia. Study on related problems in minimal surface modelling [D]. Dalian University of Technology.2013.

[2] Zhu Yaguang. Research on Geometric Modeling Method of Complex Minimal Surface [D]. Hangzhou Dianzi University, 2017.

[3] Winfried Nerdinger(Ed.)Frei Otto Complete Works:Lightweight Construction,Natural Design[M].China Architecture \& Building Press.2010.

[4] Qian Wang, From Technology to Design, 2019, Southeast University.

[5] Anonymous. Taichung Metropolitan Opera House [J]. Architectural Creation,2014 (1) : 80-125.

[6] Fornes Marc. Double Agent White[C]. ACADIA 14projects, Los Angeles,23-25 October, 2014, pp. 153164.

[7] Mariana Popescu, Matthias Rippmann, Andrew Liew, Lex Reiter,Robert J. Flatt,Tom Van Mele,Philippe Block. Structural design, digital fabrication and construction of the cable-net and knitted formwork of the KnitCandela concrete shell [J]. Structures, March,2020.

[8] Xu J., Zhang Y.;Zhao Y., Zhang X.. Data-Optimizing on Minimal Surface Pavillions: Analysis of the Whole Process of Design and Optimization of the Minimal Surface Pavillion Series "Flora" [C]. Proceedings of the 2019 DigitalFUTURES, Singapore,2019, pp. 247-256.

[9] Minimal surface. Urban Environmental Design,2020(03):211.

[10] (Japan) Kawaguchi Wei, A Bu You, et al. Mysteries of Architectural Structure: Transmission and Form of Force [M]. Translated by WANG Xiao-dun et al. Tsinghua University Press, 2012.06.

[11] LI Biao, HUA Hao. Teaching Research of Building Numerical Control Generation Technology "ANGLE_X" [J]. Journal of Architecture, 2010(10): 24-28.

[12] XU Wei-guo. Digital Construction [J]. Acta Architecture Sinica, 2009,017 (01):61-68

[13] Michalatos, P.. Design Signals: The Role of Software Architecture and Paradigms in Design Thinking and Practice[J]. Archit. Design, September, 2016(v86), pp. 108-115.

[14] WU Jun. The Beauty of Mathematics [M]. People's Posts and Telecommunications Press, 2012.

[15] XU Wei-guo. Digital Construction [J]. Acta Architecture Sinica, 2009,017 (01):61-68

[16] David P. Billington. Tower and Bridge: New Art of Structural Engineering [M]. Science Popularized Press, 1991.

[17] JIANG Zhen-yan. Parametric Generation and Design of Minimal Surfaces [D]. Nanjing University, 2018.

[18] YUAN Feng. Research on Digital Structure Performance Formation [J]. Journal of West China Human Settlement Environment, 2014, 029(006):6-12. 